\title{
Deuteron breakup effects in transfer reactions using a Weinberg state expansion method
}

\author{
A. Laid, J. A. Tostevin, and R. C. Johnson \\ Department of Physics, University of Surrey, Guildford, Surrey, GU2 5XH, United Kingdom
}

(Received 5 March 1993)

\begin{abstract}
At medium energies the distorted-wave Born approximation provides a much less reliable description of transfer reactions induced by composite projectiles. While use of the Johnson-Soper adiabatic method includes the dominant contributions from three-body breakup channels, recent experimental data have suggested the need for additional theoretical refinements. The use of Weinberg or Sturmian states provides a framework within which the adiabatic theory can be improved systematically. The results of the Weinberg states method are presented for the ${ }^{66} \mathrm{Zn}(d, p){ }^{67} \mathrm{Zn}$ (g.s.; $5 / 2^{-} ; \ell=3$ ) reaction at $88.2 \mathrm{MeV}$ incident energy. The implementation of the method is shown to reduce the three-body transfer reaction calculation to a very small coupled-channels problem. For the reaction in question, the method introduces significant corrections to the adiabatic method which agree qualitatively with earlier calculations using the quasiadiabatic method.
\end{abstract}

PACS number(s): 24.10.-i, 24.10.Eq, 24.50.+g, 25.45.Hi

\section{INTRODUCTION}

We study breakup effects on transfer reactions by use of the $(d, p)$ reaction, the simplest theoretical context in which these studies can be made. The distorted-wave Born approximation (DWBA), by construction, does not account for breakup effects and its accuracy and validity for the deuteron-nucleus system has been the subject of a large number of three-body $(n+p+$ target $)$ studies. An extensive review can be found in Ref. [1]. It is now accepted that the DWBA needs to be extended to include breakup effects. What we learn from deuteron-induced reactions is of course relevant to the study of analogous effects in reactions induced by other loosely bound cluster nuclei such as ${ }^{6} \mathrm{Li},{ }^{7} \mathrm{Li},{ }^{11} \mathrm{Be}$. In this work, we consider specifically deuteron stripping reactions at incident energies $E_{d} \approx 100 \mathrm{MeV}$, at which energies one need not include breakup effects due to the Coulomb interaction.

The Johnson-Soper adiabatic approximation [2], in which the center of mass energy of the $n$ - $p$ pair in the breakup states is assumed degenerate with the elastic channel, has provided systematic improvements over the DWBA in the description of transfer reaction angular distributions $[3,4]$. More recent, complete and accurate data on stripping and pickup transitions, with large transferred neutron angular momentum $\ell$ and total angular momentum $j=\ell-1 / 2$, at medium energies are, however, not even qualitatively reproduced [5]. As was discussed in Refs. $[5,6]$, the spin structure and dynamics of the $(d, p)$ reaction at these energies lead to the reactions being far-side dominated and to near-side/far-side interference which is a sensitive probe of high energy $n-p$ relative motion components in the three-body wave function. This reveals itself through oscillations in the measured angular distributions of certain of the spin observables. Such data provide a severe test of the quality of threebody descriptions of reaction processes.

This has resulted in a reassessment of the applicability of the adiabatic prescription. Recent calculations [6] using the quasiadiabatic approximation [7], where the $n-p$ center of mass energy in breakup configurations is assigned some average breakup energy, have yielded significant corrections to the adiabatic model in such transitions. Although the quasiadiabatic calculations of Ref. [6] lead to an improved description of the measured observables, theoretically there is ambiguity as to the correct choice of the average breakup energy inherent in the method.

Extensive three-body studies have also been carried out using the coupled discretized continuum channels (CDCC) treatment of breakup effects $[1,13]$ which has been developed to a very sophisticated level. In this case the three-body breakup continuum is discretized into a finite set of representative square integrable states $\tilde{\phi}_{i}$ in the continuum. This approach can be used as the basis of a general approach to elastic scattering, breakup, and transfer reaction studies.

In this paper we implement an alternative theoretical approach, the Weinberg state method of Johnson and Tandy [8], designed specifically for the investigation of corrections to the Johnson-Soper treatment for transfer reactions. While formulated many years ago, this procedure has not previously been investigated quantitatively. We apply the Weinberg state method to the $(d, p)$ reaction to assess the magnitude of the corrections to the adiabatic approximation and to gain some insight into the reliability of the earlier quasiadiabatic calculation predictions. We apply the method here to the ${ }^{66} \mathrm{Zn}(d, p){ }^{67} \mathrm{Zn}$ (g.s.; $5 / 2^{-} ; \ell=3$ ) reaction at $88.2 \mathrm{MeV}$ incident deuteron energy, the focus of a recent quasiadiabatic study [6].

In Sec. II we outline the formal aspects of the Weinberg state method and in Sec. III discuss its numerical implementation. In Sec. IV we compare the calculated Weinberg state predictions with those of the adiabatic and quasiadiabatic approaches. A summary and concluding remarks are presented in Sec. V. 


\section{THE WEINBERG STATE MODEL}

A detailed formulation of the Weinberg state model has been presented by Johnson and Tandy [8]. We shall only introduce the important physical and notational elements here.

\section{A. The three-body model}

The three-body wave function describing an incident deuteron beam of center of mass (c.m.) kinetic energy $E_{d}$ and binding energy $\epsilon_{d}$, will be written $\psi^{(+)}(\mathbf{r}, \mathbf{R})$, and satisfies the Schrödinger equation $\left(E=E_{d}-\epsilon_{d}\right)$

$$
\begin{aligned}
{\left[E+i \epsilon-h_{n p}-T_{R}-U(\mathbf{r}, \mathbf{R})\right] \psi^{(+)} } & (\mathbf{r}, \mathbf{R}) \\
& =i \epsilon \phi_{d}(\mathbf{r}) e^{i \mathbf{K} \cdot \mathbf{R}}
\end{aligned}
$$

where the incident boundary condition of a deuteron, with internal wave function $\phi_{d}(\mathbf{r})$ and Hamiltonian $h_{n p}$ ( $\left.=T_{r}+V_{n p}\right)$, with incident momentum $\mathbf{K}$ is made explicit through the use of a small positive quantity $\epsilon$ which will eventually be set to zero. Here $T_{r}$ and $T_{R}$ are the kinetic energy operators for the $n-p$ relative and c.m. motion. The interaction $U(\mathbf{r}, \mathbf{R})$ to be used in Eq. (1) has been discussed extensively by many authors $[2,9,8]$. Throughout this work we assume the interaction to be the sum of the neutron- and proton-target phenomenological optical potentials evaluated at half the incident deuteron energy. The proton target Coulomb interaction is assumed to act on the c.m. of the $n-p$ system. is $[8]$

The $(d, p)$ stripping amplitude in the three-body model

$$
T_{d p}=\left\langle\chi^{(-)}\left(\mathbf{r}_{p}\right) \Phi_{\ell j}\left(\mathbf{r}_{n}\right)\left|V_{n p}\right| \psi^{(+)}\right\rangle,
$$

where $\Phi$ describes the transferred neutron-core bound state and $\chi^{(-)}$, the proton distorted wave, describes proton elastic scattering from the residual nucleus.

Since the $n-p$ interaction is short ranged, an accurate evaluation of $T_{d p}$ requires that $\psi^{(+)}(\mathbf{r}, \mathbf{R})$ be calculated accurately only in the neighborhood of $|\mathbf{r}|=0$, i.e., $|\mathbf{r}| \approx$ range of $V_{n p}$. It is well known that the wave function $\psi^{(+)}(r=0, \mathbf{R})$ satisfies much simpler boundary conditions than does the full three-body wave function, and the solution can be reduced to an effective two-body problem. This simplification for small $|\mathbf{r}|$ is also inherent in the Weinberg state method in the sense that, for a given $n-p$ interaction $V_{n p}$, it is possible to develop an effective two-body method for the projected state $V_{n p}\left|\psi^{(+)}\right\rangle$. Since this projection appears explicitly in the $(d, p)$ transition amplitude, Eq. (2), the Weinberg state technique is well adapted to an accurate determination of the transfer amplitude.

\section{B. The Weinberg state expansion}

For the calculation of $V_{n p}\left|\psi^{(+)}\right\rangle$, Johnson and Tandy expand the three-body wave function in a discrete set of states, complete on the range of $V_{n p}$, namely, the Weinberg [10] eigenstates $\phi_{i}$ defined by the eigenvalue equation

$$
\left[-\epsilon_{d}-T_{r}-\alpha_{i} V_{n p}\right] \phi_{i}(\mathbf{r})=0, \quad i=1,2, \ldots,
$$

with normalizations

$$
\left\langle\phi_{i}\left|V_{n p}\right| \phi_{j}\right\rangle=-\delta_{i j}
$$

The three-body wave function is thus expanded,

$\psi^{(+)}(\mathbf{r}, \mathbf{R})=\sum_{i=1}^{\infty} \phi_{i}(\mathbf{r}) \chi_{i}^{(+)}(\mathbf{R}),|\mathbf{r}| \leq$ range of $V_{n p}$

where, using Eq. (4),

$$
\chi_{i}^{(+)}(\mathbf{R})=-\left\langle\phi_{i}\left|V_{n p}\right| \psi^{(+)}\right\rangle .
$$

The only physical state among the $\phi_{i}$ is the first $(i=$ $1, \alpha_{1}=1$ ), which is proportional to the deuteron ground state. The $\alpha_{i}$ increase monotonically with $i$ and are obtained by solution of the eigenvalue problem, Eq. (3). Since, with increasing $i$ and $\alpha_{i}$, the radial behavior of the $\phi_{i}$ become increasingly oscillatory within the range of $V_{n p}$, the higher $\phi_{i}$ states are associated with higher energy relative $n-p$ configurations. For any particular reaction system there will be an upper limit to the physical $n-p$ continuum energies which contribute significantly to $V_{n p}\left|\psi^{(+)}\right\rangle$and the reaction process. Thus, in practical applications, a truncation of the sum over Weinberg states to some number $N$ of terms will be expected. This truncation will be dictated by the requirement of accurate numerical calculations. Introducing such a truncated expansion, the coupled equations obtained for the $\chi_{i}^{(+)}$are

$$
\left[E_{d}+i \epsilon-T_{R}\right]\left|\chi_{i}^{(+)}\right\rangle=i \epsilon \delta_{i 1} N_{d}|\mathbf{K}\rangle+\sum_{j=1}^{N} U_{i j}(\mathbf{R})\left|\chi_{j}^{(+)}\right\rangle
$$

where $N_{d}$ is the constant

$$
N_{d}=-\left\langle\phi_{1}\left|V_{n p}\right| \phi_{d}\right\rangle
$$

and

$$
\begin{gathered}
U_{i j}(\mathbf{R})=V_{i j}(\mathbf{R})+C_{i j} \\
V_{i j}(\mathbf{R})=-\left\langle\phi_{i}\left|V_{n p} U(\mathbf{r}, \mathbf{R})\right| \phi_{j}\right\rangle, \\
C_{i j}=\beta_{i j}\left(\alpha_{j}-1\right)
\end{gathered}
$$

with

$$
\beta_{i j}=\left\langle\phi_{i}\left|V_{n p}^{2}\right| \phi_{j}\right\rangle
$$

The coupling potentials $U_{i j}$ thus represent the interactions coupling the internal $n-p$ states $\phi_{i}$ and $\phi_{j}$ and contain two contributions. The $V_{i j}$ are the contributions due 
to the nucleon-target interactions. There are also, however, constant coupling terms $C_{i j}$ which arise because the Weinberg basis states are not eigenfunctions of the $n-p$ Hamiltonian $h_{n p}$. Explicitly, the required matrix elements of $h_{n p}$ are

$$
\left\langle\phi_{i}\left|V_{n p} h_{n p}\right| \phi_{j}\right\rangle=-\left[\beta_{i j}\left(\alpha_{j}-1\right)-\epsilon_{d} \delta_{i j}\right],
$$

with off diagonal contributions which induce transitions between the Weinberg states.

A characteristic of the coupled equations (7) is that only the $i=1$ channel solution contains the elastic component, but every channel $(i=1,2, \ldots, N)$ explicitly includes breakup effects. Furthermore, retaining only the first term in the expansion leads to the adiabatic equation $[2,4]$

$$
\left[E_{d}+i \epsilon-T_{R}-V_{11}(\mathbf{R})\right]\left|\chi_{1}^{(+)}\right\rangle=i \epsilon N_{d}|\mathbf{K}\rangle .
$$

Thus the adiabatic approximation appears as the solution of lowest order $N=1$. In this sense, an expansion comprising several Weinberg states is expected to provide an improved description of higher $n-p$ relative energy components which are not included accurately in the original adiabatic method.

For any $N$, the conditions $\phi_{1} \propto \phi_{d},\left\langle\phi_{i}\left|V_{n p}\right| \phi_{j}\right\rangle=-\delta_{i j}$ guarantee that $\chi_{1}^{(+)}$satisfies the Johnson-Soper equation, but only when all other $\chi_{i}$ are neglected. A purpose of this paper is to investigate these other components. To do this one has to handle the couplings produced by $\left\langle\phi_{i}\left|V_{n p} h_{n p}\right| \phi_{j}\right\rangle$. These couplings have infinite range in $R$. It is desirable therefore to use a set of basis states for which this coupling matrix is diagonal since then the coupled equations for the $\chi_{i}$ have standard form with finite range couplings. The Weinberg states form a natural choice for such a basis set as they are a complete set in a space with a metric with weight factor $V_{n p}$. Choosing Weinberg states based on the energy $-\epsilon_{d}$ guarantees $\phi_{1} \propto \phi_{d}$.

\section{Removal of the constant coupling terms}

From the calculational point of view, the coupledchannels equations, as expressed in Eq. (7), are not in a standard or useful form, due to the infinite ranged couplings implied by the constant terms $C_{i j}$. As was shown in detail in Ref. [8], the constant coupling matrix can always be diagonalized by a suitable nonsingular and nonunitary matrix $\mathbf{A}$. We use this result and write

$$
\mathbf{C}=\mathbf{A}^{-1} \lambda \mathbf{A}
$$

with $\lambda$ a diagonal matrix, with elements $\lambda_{1}, \ldots, \lambda_{N}$ and where $\lambda_{1}=0$. The $\lambda_{i}$ depend implicitly upon the basis size $N$. The particular procedure used to calculate the $\mathbf{A}$ and $\lambda$ is outlined in the following section. Thus, after diagonalization, the coupled equations take the form

$$
\begin{aligned}
{\left[E_{d}-\lambda_{i}+i \epsilon-T_{R}\right]\left|F_{i}^{(+)}\right\rangle=} & i \epsilon \delta_{i 1} N_{d}|\mathbf{K}\rangle \\
& +\sum_{j=1}^{N} W_{i j}^{N}(\mathbf{R})\left|F_{j}^{(+)}\right\rangle
\end{aligned}
$$

where the center of mass states $\left|F_{i}^{(+)}\right\rangle$are related to the $\left|\chi_{i}^{(+)}\right\rangle$by

$$
\left|F_{i}^{(+)}\right\rangle=\sum_{j=1}^{N} A_{i j}\left|\chi_{j}^{(+)}\right\rangle
$$

The elements of the coupling matrix $\mathbf{W}^{N}=\mathbf{A V A}^{-1}$ are explicitly

$$
W_{i j}^{N}(\mathbf{R})=\left\langle\Omega_{i}^{N}\left|V_{n p} U(\mathbf{r}, \mathbf{R})\right| \Delta_{j}^{N}\right\rangle
$$

where two sets of $n-p$ relative motion states, $\left\langle\Omega_{i}^{N}\right|$ and $\left|\Delta_{j}^{N}\right\rangle$, defined by

$$
\begin{aligned}
\left\langle\Omega_{i}^{N}\right| & =\sum_{k=1}^{N} A_{i k}\left\langle\phi_{k}\right| \\
\left|\Delta_{j}^{N}\right\rangle & =\sum_{q=1}^{N}\left|\phi_{q}\right\rangle A_{q j}^{-1},
\end{aligned}
$$

have been introduced. They satisfy the orthonormality condition

$$
\left\langle\Omega_{i}^{N}\left|V_{n p}\right| \Delta_{j}^{N}\right\rangle=-\delta_{i j}
$$

There is no way to avoid the fact that the three-body Schrödinger equation couples all regions of $r$ together. At the differential equation level, for a local $V_{n p}$ and $U(\mathbf{r}, \mathbf{R})$, this coupling occurs through the derivatives in $T_{r}$ and it is the diagonalization of this operator which is involved in the $C_{i j}$ of Eq. (9). Switching to the $\left\langle\Omega_{i}^{N}|,| \Delta_{i}^{N}\right\rangle$ basis is one way of handling this coupling. The quantitative effects of this coupling and the way they are influenced by the properties of $U(\mathbf{r}, \mathbf{R})$ now appear through the coupling matrix $W_{i j}^{N}$. This is natural because, if the $r$ dependence of $U(\mathbf{r}, \mathbf{R})$ was negligible over the range of $V_{n p}$, the matrix $W_{i j}^{N}$ would be diagonal and the effects due to the coupling of different $r$ regions would be irrelevant. In this limit $U$ would not generate any breakup.

From Eq. (16), the elements $\lambda_{i}$ of the diagonal array $\lambda$ are seen to play the role of the excitation energies of the modified internal states $\left|\Delta_{i}^{N}\right\rangle$ of the $n$-p system. In terms of these internal states the three-body wave function is

$$
\psi^{(+)}(\mathbf{r}, \mathbf{R})=\sum_{i=1}^{N} F_{i}^{(+)}(\mathbf{R}) \Delta_{i}^{N}(\mathbf{r})
$$

and hence

$$
F_{i}^{(+)}(\mathbf{R})=-\left\langle\Omega_{i}^{N}\left|V_{n p}\right| \psi^{(+)}\right\rangle
$$

The $\left|\Delta_{i}^{N}\right\rangle$ for $i>1$ thus play the role of effective inelastic states of the $n-p$ system, for $|\mathbf{r}| \leq$ range of $V_{n p}$, with excitation energy $\lambda_{i}$ above the deuteron ground state while $\left|\Delta_{1}^{N}\right\rangle=\left|\phi_{1}\right\rangle$. Upon division of Eq. (16) by $N_{d}$, the coupled equations can be solved for the $\left|F_{i}^{(+)} / N_{d}\right\rangle$ subject to the normal boundary conditions of a deuteron (actually state $\phi_{1}$ ) incident in the $i=1$ channel. It should be noted, however, that, although the coupled 
equations are now of a conventional form in the sense of finite ranged coupling interactions, these coupling terms are not symmetric, as is usually the case with such coupled-channels sets in nuclear physics applications, i.e., $W_{i j}^{N}(\mathbf{R}) \neq W_{j i}^{N}(\mathbf{R})$.

\section{NUMERICAL IMPLEMENTATION}

In this work we construct the Weinberg basis set $\phi_{i}$ using a central $n$ - $p$ interaction. The nucleon-target optical potentials will also be assumed to be spin independent and only the relative $S$-wave continuum of the $n-p$ system is included.

\section{A. The Hulthén Weinberg basis}

We construct the Weinberg basis from the Hulthén $n-p$ interaction

$$
V_{n p}^{H}(r)=V_{0} /\left(e^{\beta r}-1\right)
$$

where, to bind the deuteron ground state with energy $\epsilon_{d}=\hbar^{2} \kappa^{2} / 2 \mu_{n p}$,

$$
V_{0}=-\frac{\hbar^{2} \beta^{2}}{2 \mu_{n p}}\left(1+\frac{2 \kappa}{\beta}\right)
$$

where $\mu_{n p}$ is the $n$ - $p$ reduced mass. For the present exploratory investigation, this choice of potential is particularly convenient since the $S$-state Weinberg solutions $\phi_{i}(r)$ can then be expressed analytically [11]. We use the notation that $\phi_{i}(r)=\left[S_{i}(r) / r\right] Y_{00}(\hat{r})$, with $Y_{00}$ the spherical harmonic. In the Hulthén case the Weinberg state parameters $\alpha_{i}$, Eq. (3), are given by

$$
\alpha_{i}=i\left(\frac{2 \kappa+i \beta}{2 \kappa+\beta}\right)
$$

and the radial form of the Weinberg states $S_{i}(r)$ can be written

$$
S_{i}(r)=e^{-\kappa r} \sum_{j=0}^{i} a_{j}^{(i)} e^{-j \beta r}, \sum_{j=0}^{i} a_{j}^{(i)}=0
$$

where the $a_{j}^{(i)}$ satisfy the recurrence relation $a_{0}^{(i)} \neq 0$,

$$
a_{j+1}^{(i)}=a_{j}^{(i)}\left(\frac{j \omega_{1}-\omega_{i}+j(j-1)}{(j+1)\left(j+\omega_{1}\right)}\right),
$$

with

$$
\omega_{i}=-\alpha_{i}\left(\frac{2 \mu_{n p} V_{0}}{\hbar^{2} \beta^{2}}\right)
$$

While the normalization condition on the $a_{j}^{(i)}$, such that $\left\langle\phi_{i}\left|V_{n p}^{H}\right| \phi_{i}\right\rangle=-1$, and the matrix elements $\beta_{i j}=$ $\left\langle\phi_{i}\left|\left[V_{n p}^{H}\right]^{2}\right| \phi_{j}\right\rangle$ can also be expressed analytically the resulting sums contain large and canceling contributions. The normalizations and calculation of the matrix ele- ments were found to be more accurately calculated by direct numerical integration of the radial functions.

As noted earlier the $i=1$ solution is proportional to the deuteron ground state, i.e., $\phi_{d}(r)=N_{d} \phi_{1}(r)$, where

$$
\phi_{d}(r)=\mathcal{N} e^{-\kappa r}\left[1-e^{-\beta r}\right] Y_{00}(\hat{r}) / r
$$

with $\mathcal{N}$ the usual Hulthén $S$-state normalization constant, $\mathcal{N}=\sqrt{2 \kappa(\kappa+\beta)(2 \kappa+\beta)} / \beta$. We take the parameters $\epsilon_{d}=2.226 \mathrm{MeV}, \beta=5.255 \kappa$, for which $V_{0}=-84.86$ $\mathrm{MeV}$ and $N_{d}=4.02 \mathrm{MeV}^{1 / 2}$. The radial behavior of the first three $S_{i}(r)$ are presented in Fig. 1 together with, on the same scale, the Hulthén $V_{n p}^{H}(r)$ to display the behavior of the states within the radial extent of the $n-p$ interaction.

\section{B. Diagonalization of the coupling matrix}

For the calculation of the transformation matrix $\mathbf{A}$, Eq. (15), we make use of the analysis of [8]. Rather than attempt a direct diagonalization of the large unbalanced and unsymmetric $N \times N$ coupling matrix $\mathbf{C}$, where

$$
C_{i j}=\beta_{i j}\left(\alpha_{j}-1\right),
$$

we make use of the fact that $\mathbf{C}$ can be written

$$
\mathbf{C}=\alpha^{-1} \mathbf{B} \alpha
$$

Here $\alpha$, a diagonal matrix, has elements $\left(1,\left(\alpha_{2}-\right.\right.$ $\left.1)^{1 / 2}, \ldots,\left(\alpha_{N}-1\right)^{1 / 2}\right)$ and $\mathbf{B}$ is of the form

$$
\mathbf{B}=\left(\begin{array}{ll}
0 & \mathbf{x} \\
0 & \mathbf{b}
\end{array}\right),
$$

with $\mathbf{b}$ the $(N-1) \times(N-1)$ matrix with elements

$$
b_{i j}=\left(\alpha_{i}-1\right)^{1 / 2} \beta_{i j}\left(\alpha_{j}-1\right)^{1 / 2},
$$

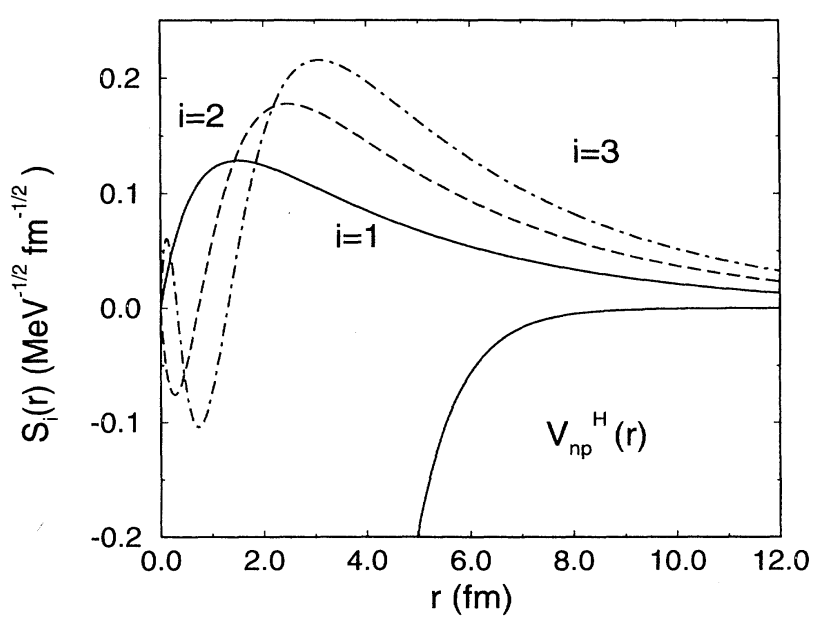

FIG. 1. Calculated radial $S$-wave Weinberg states $S_{i}(r)$ $(i=1,2,3)$ using the Hulthén $n-p$ interaction of Eq. (24). The actual Hulthén potential is also shown on the same vertical scale (then in $\mathrm{MeV}$ ). 
and $\mathbf{x}$ the $(N-1)$ component row with elements $\left(\beta_{12}\left(\alpha_{2}-\right.\right.$ $\left.1)^{1 / 2}, \ldots, \beta_{1 N}\left(\alpha_{N}-1\right)^{1 / 2}\right)$.

Since by construction the submatrix $\mathbf{b}$ is real and symmetric, it can always be diagonalized by a similarity transformation and its $(N-1)$ real eigenvalues $\lambda_{i}$, $(i=2, \ldots, N)$ and normalized eigenvectors $\mathbf{v}^{(i)}$ are readily calculated with high precision. It follows also that the matrix $\mathbf{B}$ is similar to a triangular matrix whose diagonal elements are $\left(\lambda_{1}=0, \lambda_{2}, \ldots, \lambda_{N}\right)$ and which are therefore the $N$ distinct eigenvalues of $\mathbf{B}$.

Thus, for each eigenvalue $\lambda_{i}(i=2, \ldots, N)$, for which $\mathbf{b v}^{(i)}=\lambda_{i} \mathbf{v}^{(i)}$, the vector

$$
\mathbf{V}^{(i)}=\left(\begin{array}{l}
V_{1}^{(i)} \\
\mathbf{v}^{(i)}
\end{array}\right)
$$

will be an eigenvector of $\mathbf{B}$ with the same eigenvalue provided that $V_{1}^{(i)}=\mathbf{x} \mathbf{v}^{(i)} / \lambda_{i}$. Since the $\mathbf{v}^{(i)}$ are constructed to be normalized, it follows that the normalized eigenvectors of $\mathbf{B}$, denoted $\mathcal{V}^{(i)}$, are $(i=2, \ldots, N)$

$$
\mathcal{V}^{(i)}=\frac{1}{\sqrt{1+\left[V_{1}^{(i)}\right]^{2}}}\left(\begin{array}{c}
V_{1}^{(i)} \\
\mathbf{v}^{(i)}
\end{array}\right), \quad V_{1}^{(i)}=\frac{\mathbf{x v}^{(i)}}{\lambda_{i}} .
$$

In the case $i=1$ then

$$
\mathcal{V}^{(1)}=\left(\begin{array}{l}
1 \\
\mathbf{0}
\end{array}\right)
$$

with eigenvalue $\lambda_{1}=0$. It follows therefore, constructing the matrix $\mathbf{D}$, whose columns are the $\mathcal{V}^{(i)}$ in increasing order of $i$, that

$$
\mathbf{B}=\mathbf{D}^{-1} \lambda \mathbf{D}
$$

and therefore that the required transformation matrix $\mathbf{A}=\mathbf{D} \alpha$, since

$$
\mathbf{C}=\alpha^{-1} \mathbf{B} \alpha=\alpha^{-1}\left[\mathbf{D}^{-1} \lambda \mathbf{D}\right] \alpha=\mathbf{A}^{-1} \lambda \mathbf{A} \text {. }
$$

The calculated elements $\lambda_{i}$ and elements of $\mathbf{A}$ were found to be accurately reproduced when using a number of available matrix eigenvalue and eigenvector library routines.

\section{REACTION CALCULATIONS}

We apply the techniques developed above for the calculation of the three-body wave function, Eq. (22), and of reaction observables in the ${ }^{66} \mathrm{Zn}(d, p){ }^{67} \mathrm{Zn}$ (g.s.; $5 / 2^{-} ; \ell$ =3) transition at $88.2 \mathrm{MeV}$ incident deuteron energy. Analysis of the high quality spin polarization data [6] for this transition have shown that the adiabatic approximation, the $N=1$ limit of the Weinberg state expansion, provides an inadequate description of the threebody breakup channels. The spin structure (large $\ell$, $j=\ell-1 / 2)$ and dynamics of the $(d, p)$ reaction at such energies $[5,6]$ lead to the reaction being far-side dominated and to a near-side/far-side interference which is a sensitive probe of high $n-p$ relative momentum com- ponents in the wave function and is manifest through oscillations in the angular distributions of the measured spin observables. These oscillations were able to be reproduced qualitatively using the quasiadiabatic technique [6] for a reasonable choice of the mean breakup energy as input.

Since in the present analysis we do not include the non$S$-wave breakup continuum, the deuteron ground state $D$-wave component, or the nucleon-target spin-orbit interactions, important ingredients quantitatively, we cannot compare the theoretical calculations directly with the experimental data. Our aim here is to investigate the nature of the convergence of the calculated wave functions and observables with the size of the Weinberg basis set $N$ and the qualitative effects of these nonadiabatic $(N>1)$ contributions upon the calculated cross section and vector analyzing power $i T_{11}$ observables. We will compare these Weinberg state expansion (WSE) calculations with those of the adiabatic and quasiadiabatic methods using the same $n-p$ interaction and nucleon-target interaction inputs.

\section{A. The modified Weinberg basis}

Given the diagonalization procedure of Sec. III we can now calculate the representative continuum energies $\lambda_{i}$ and the matrix $\mathbf{A}$. Given $\mathbf{A}$, we can construct the new basis sets $\left\langle\Omega_{i}^{N}\right|$, Eq. (19), and $\left|\Delta_{i}^{N}\right\rangle$, Eq. (20), for any value of $N$. We first investigate the spectrum $\lambda_{i}$ of relative $n-p$ energies as a function of $N$. This part of the analysis is clearly a function only of the chosen $n-p$ interaction and is independent of any particular reaction system. As is also clear from the coupled equations, Eq. (16), when applied to a specific reaction, with deuteron incident kinetic energy $E_{d}$, the effective channel c.m. energies are $E_{i}=E_{d}-\lambda_{i}$ and the magnitude of the calculated $\lambda_{i}$ will determine whether a given internal state $\left|\Delta_{i}^{N}\right\rangle$ is open $\left(E_{i}>0\right)$ or closed $\left(E_{i}<0\right)$ asymptotically $(R \rightarrow \infty)$.

In Fig. 2 we show the lowest three calculated energy eigenvalues $\left(\lambda_{1}=0\right) \lambda_{i}(i=2,3,4)$, as a function of the size of the original Weinberg basis $\phi_{i}$, for $N$ values from 1 to 35 . The calculated $\lambda_{i}$ obtained in this work are collected in Table I for Weinberg state bases $N \leq 35$. Clear immediately from the figure and table is that, of the $N-1$ nonzero $\lambda_{i}$, only three have values below $200 \mathrm{MeV}$ for $N \leq 35$. Thus, in the context of deuteron-induced reactions with $E_{d} \approx 100 \mathrm{MeV}$, of interest here, for basis sizes $N \leq 15$ there will be only two open channels $\left|\Delta_{1}^{N}\right\rangle$ and $\left|\Delta_{2}^{N}\right\rangle$, the third channel being increasingly closed as $N$ is reduced. For basis sizes $N>15$ then the third state $\left|\Delta_{3}^{N}\right\rangle$ is also open. At $N=35$ the fourth channel remains closed by some $45 \mathrm{MeV}$, corresponding to broken up $n-p$ fragments with relative energies of order $130 \mathrm{MeV}$.

We normalize these relative motion states such that

$$
\left\langle\mathbf{r} \mid \Delta_{i}^{N}\right\rangle=\Delta_{i}^{N}(r) Y_{00}(\hat{r})
$$

and similarly

$$
\left\langle\Omega_{i}^{N} \mid \mathbf{r}\right\rangle=\Omega_{i}^{N}(r) Y_{00}(\hat{r})
$$




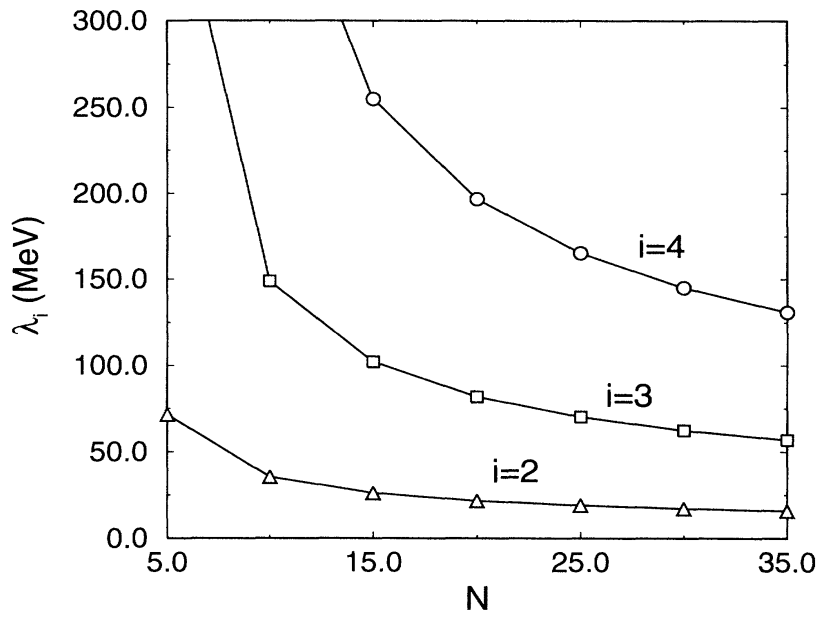

FIG. 2. Calculated energy spectrum $\lambda_{i}(i=2,3,4)$ of the $n-p$ states $\left|\Delta_{i}^{N}\right\rangle$ as a function of the size of the Weinberg state basis $N$ for the Hulthén $n-p$ interaction of Eq. (24).

The radial behavior $r \Delta_{i}^{N}(r)$ of the open $n$ - $p$ relative motion states $\left|\Delta_{i}^{N}\right\rangle(i=1,2,3)$ are presented in Fig. 3 in the case that $N=35$. The radial forms $r \Omega_{i}^{N}(r)$ of the corresponding states $\left\langle\Omega_{i}^{N}\right|(i=1,2,3)$, required for the calculation of the coupling potentials $W_{i j}^{N}$, Eq. (18), are presented in Fig. 4, also for $N=35$. The relative magnitudes of the two sets of states should be noted. The orthonormality relation, Eq. (21), was satisfied to high accuracy by these calculated $n-p$ states.

\section{B. The coupling interactions}

As was stated earlier, we assume that the nucleonnucleus optical potentials are purely central. In the subsequent transfer reaction calculations the spin-orbit component is neglected in the exit channel also. We use for the entrance channel nucleon-nucleus optical potential the global parameter set of Becchetti and Greenlees [12] with energy $E_{d} / 2$. As was shown above, for original basis sizes $N \leq 35$, only a very small number $n \leq 3$ of the transformed basis states $\left|\Delta_{i}^{N}\right\rangle$ are open channels. For $N=35$, the calculated real and imaginary parts of a typical diagonal continuum to continuum interaction, $W_{22}^{N}(R)$, and a ground state to continuum and a continuum to continuum off diagonal coupling, $W_{21}^{N}(R)$ and $W_{23}^{N}(R)$, term are presented in Fig. 5 . In all cases, the presence of the short ranged $n-p$ interaction in the coupling potential matrix elements means that the range of all terms is essentially that of the range of the nucleon-

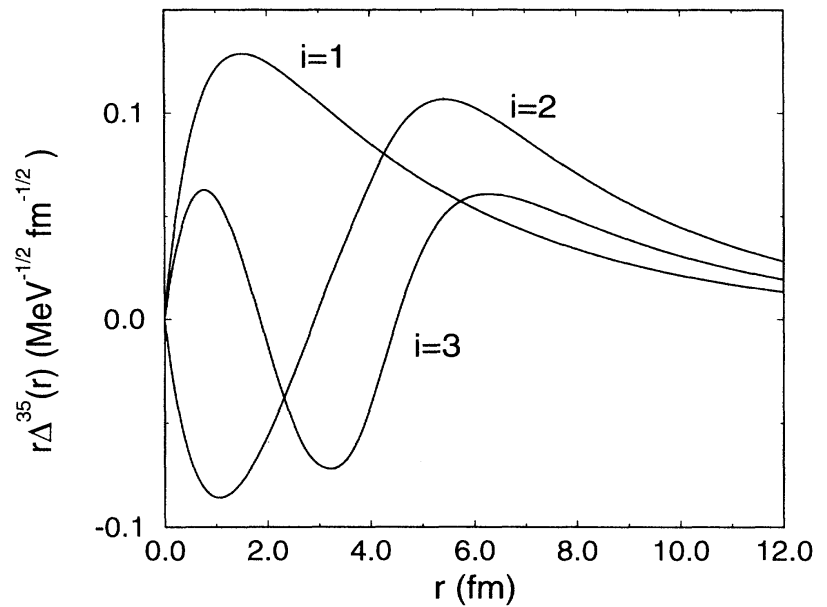

FIG. 3. Calculated radial form of the $n$ - $p$ states $r \Delta_{i}^{35}(r)$ $(i=1,2,3)$ for the Hulthén $n-p$ interaction.

target interactions. The coupling interactions of the WSE are thus characteristically different to the longer range matrix elements $\left\langle\tilde{\phi}_{i}|U(\mathbf{r}, \mathbf{R})| \tilde{\phi}_{j}\right\rangle$ which enter the CDCC treatment of breakup effects $[1,13]$ where the folding integral is over the much longer ranged continuum bin states $\left|\hat{\phi}_{j}\right\rangle$.

\section{Solution of the coupled equations}

Given the set of coupling interactions $W_{i j}^{N}(R)$ the coupled-channels equations for the $F_{i}^{(+)}(\mathbf{R})$ are readily solved for any size of original Weinberg basis set $N$ and for the inclusion of any number $n \leq N$ of transformed basis states $\Delta_{i}^{N}(r)$. Although not explicitly indicated, it must be remembered that the states $F_{i}^{(+)}$depend implicitly upon the choices made for $N$ and $n$. Such sets of coupled equations are frequently encountered in nuclear physics, hence the existence of several dedicated computer codes. The code FRESCO [14] was used in this work since it incorporates the option to read nonsymmetric coupling interactions. In the present work we include only those $n-p$ states which are open, i.e., $E_{i}=E_{d}-\lambda_{i} \geq 0$. An attempt to assess the importance of this approximation is made in the following.

\section{Transfer reaction calculations}

As in Ref. [6], the ${ }^{66} \mathrm{Zn}(d, p)$ transfer reaction calculations are carried out in a zero-range approxima-

TABLE I. Calculated energies $\lambda_{i}(\mathrm{MeV})$ obtained by diagonalization of the coupling matrix $\mathbf{C}$ for size of Weinberg basis $N \leq 35$.

\begin{tabular}{lccccccc}
\hline \hline$N$ & 5 & 10 & 15 & 20 & 25 & 30 & 35 \\
\hline$\lambda_{1}$ & 0.0 & 0.0 & 0.0 & 0.0 & 0.0 & 0.0 & 0.0 \\
$\lambda_{2}$ & 71.55 & 35.49 & 26.14 & 21.69 & 19.03 & 17.23 & 15.92 \\
$\lambda_{3}$ & 404.57 & 148.77 & 102.10 & 81.75 & 70.09 & 62.42 & 56.90 \\
$\lambda_{4}$ & 1941.65 & 404.49 & 254.76 & 196.66 & 165.10 & 144.98 & 130.89 \\
\hline \hline
\end{tabular}




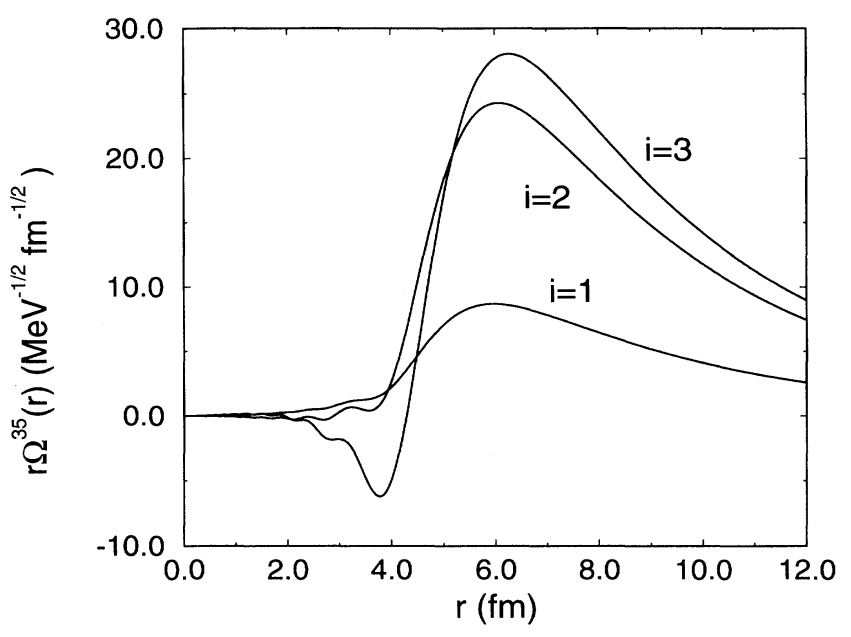

FIG. 4. Calculated radial form of the $n-p$ states $r \Omega_{i}^{35}(r)$ $(i=1,2,3)$ for the Hulthén $n-p$ interaction.

tion using the computer program TWOFNR [15]. The neutron- ${ }^{66} \mathrm{Zn}$ bound state potential parameters and the final state proton- ${ }^{67} \mathrm{Zn}$ optical potential parameters were taken from Table I of [6] with, in the case of the proton potential, the strength of the spin-orbit interaction set to zero. Using the Weinberg expansion of the three-body wave function, Eq. (22), then

$$
T_{d p} \approx \sum_{i=1}^{n}\left\langle\chi^{(-)}\left(\mathbf{r}_{p}\right) \Phi_{\ell j}\left(\mathbf{r}_{n}\right)\left|V_{n p}\right| F_{i}^{(+)}(\mathbf{R}) \Delta_{i}^{N}(\mathbf{r})\right\rangle
$$

In the zero-range approximation, the neutron- and proton-target wave functions $\Phi_{\ell j}$ and $\chi^{(-)}$are assumed to vary slowly with $\mathbf{r}$, over the range of the $n-p$ interaction, in the $(d, p)$ transition transition. Thus one writes

$$
\int d \mathbf{r} V_{n p}(r) \psi^{(+)}(\mathbf{r}, \mathbf{R})=\sum_{i=1}^{n} G_{i}^{N} F_{i}^{(+)}(\mathbf{R})
$$

where the $G_{i}^{N}$, the zero-range strengths appropriate to each open channel, are

$$
G_{i}^{N}=\sqrt{4 \pi} \int d r r^{2} V_{n p}^{H}(r) \Delta_{i}^{N}(r)
$$

The zero-range $(d, p)$ transition amplitude is therefore

$$
T_{d p}^{\mathrm{ZR}} \approx\left\langle\chi^{(-)}(\mathbf{R}) \Phi_{\ell j}(\mathbf{R}) \mid \sum_{i=1}^{n} G_{i}^{N} F_{i}^{(+)}(\mathbf{R})\right\rangle
$$

The calculated $G_{i}^{N}$ used in this work are collected in Table II for those open channels which arise for $N \leq 35$.

The description of the $n$ - $p$ c.m. motion introduced to the transfer reaction calculation in the WSE is therefore

$$
\mathcal{X}_{n}^{N}(\mathbf{R})=\sum_{i=1}^{n} G_{i}^{N} F_{i}^{(+)}(\mathbf{R})
$$

where the dependence on the basis size $N$ and the number
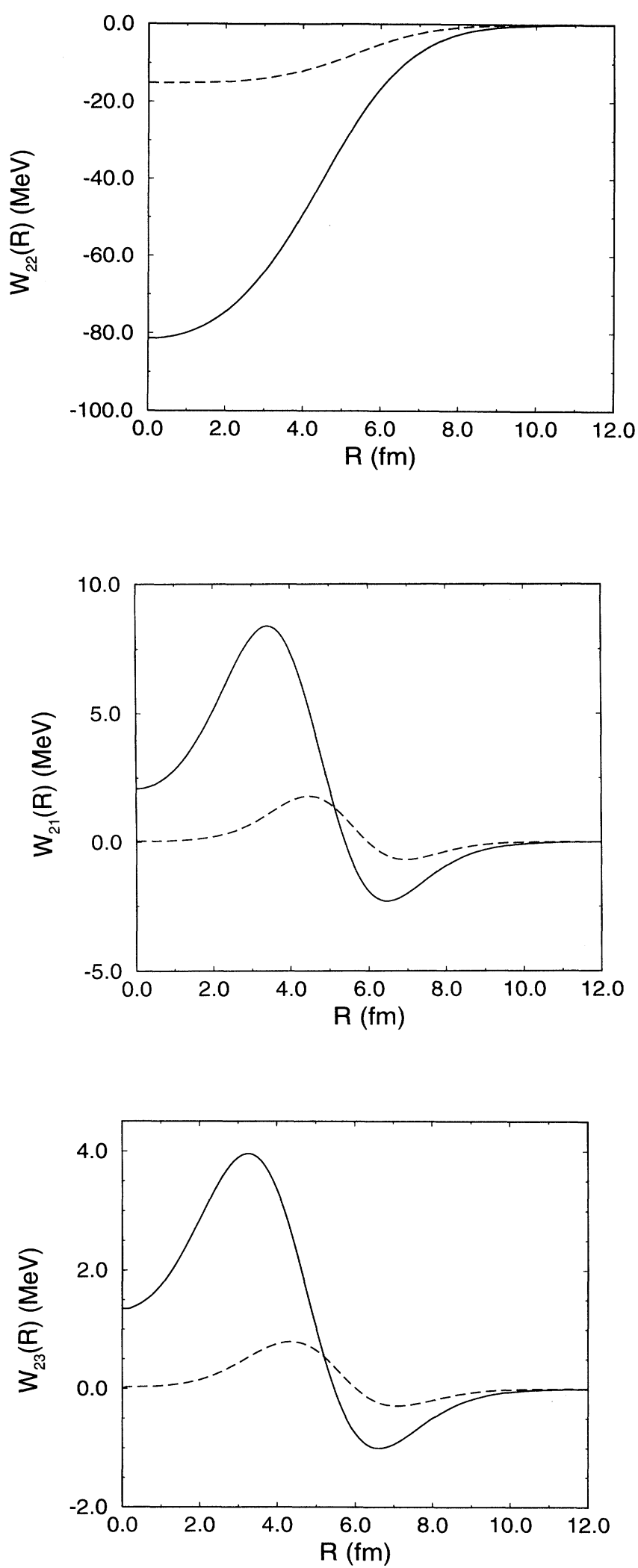

FIG. 5. Calculated real (solid lines) and imaginary (dashed lines) components of the diagonal interaction $W_{22}^{N}(R)$ in the continuum and of the ground state to continuum and continuum to continuum couplings $W_{21}^{N}(R)$ and $W_{23}^{N}(R)$ for $N=35$. 
TABLE II. Calculated zero-range normalization strengths $G_{i}^{N}\left(\mathrm{MeV}^{1 / 2} \mathrm{fm}^{3 / 2}\right)$ for the open channels in the ${ }^{66} \mathrm{Zn}(d, p)$ reaction at $88.2 \mathrm{MeV}$ for size of Weinberg basis $N \leq 35$.

\begin{tabular}{lrrrrrrr}
\hline \hline$N$ & \multicolumn{1}{c}{5} & 10 & \multicolumn{1}{c}{15} & \multicolumn{1}{c}{20} & \multicolumn{1}{c}{25} & \multicolumn{1}{c}{30} & \multicolumn{1}{c}{35} \\
\hline$G_{1}^{N}$ & -31.10 & -31.10 & -31.10 & -31.10 & -31.10 & -31.10 & -31.10 \\
$G_{2}^{N}$ & 9.21 & 12.86 & 14.41 & 15.31 & 15.90 & 16.32 & 16.64 \\
$G_{3}^{N}$ & & & & -7.01 & -7.53 & -7.95 & -8.30 \\
\hline \hline
\end{tabular}

of channels $n$ included in the calculation of the $F_{i}^{(+)}(\mathbf{R})$ is made explicit. The $n$ - $p$ c.m. motion in the JohnsonSoper adiabatic model is, in the same notation,

$$
\mathcal{X}_{1}^{1}(\mathbf{R})=G_{1}^{1} F_{1}^{(+)}(\mathbf{R})
$$

where now $F_{1}^{(+)}$is obtained by solution of Eq. (16) with $N=1$.

In Fig. 6 we compare the moduli of these entrance channel wave functions in the $L=0,7,13$, and 20 $n-p$ c.m. partial waves, obtained using the WSE model, $\mathcal{X}_{n}^{N}(\mathbf{R})(N=35, n=3)$ (solid lines), and adiabatic
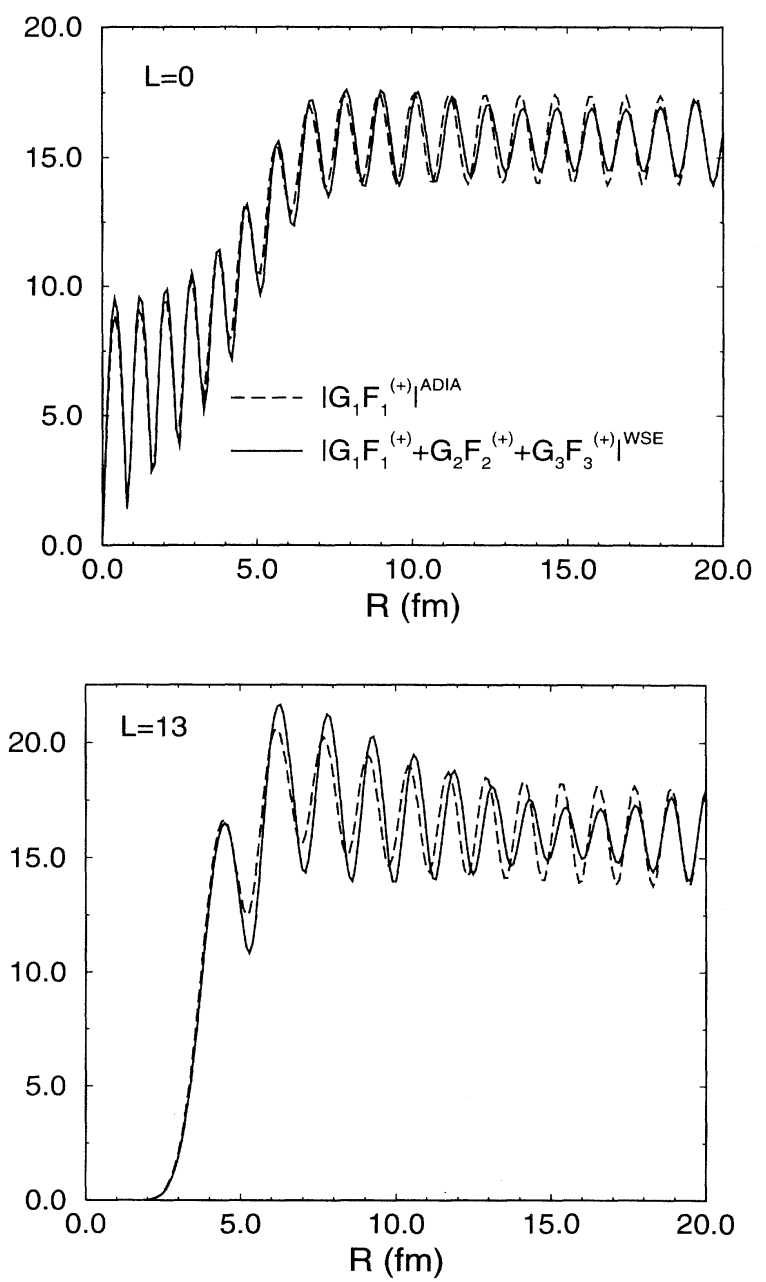

model (dashed lines). It is clear that the corrections to the adiabatic model are significant, particularly in the near-grazing partial wave $(L \approx 15)$.

\section{E. Results}

The entrance channel wave functions $\mathcal{X}_{n}^{N}(\mathbf{R})$ were read into the transfer reaction code TWOFNR and the transfer reaction overlap integrals, Eq. (44), and the reaction observables evaluated. Figure 7 shows the calculated differential cross section and vector analyz-
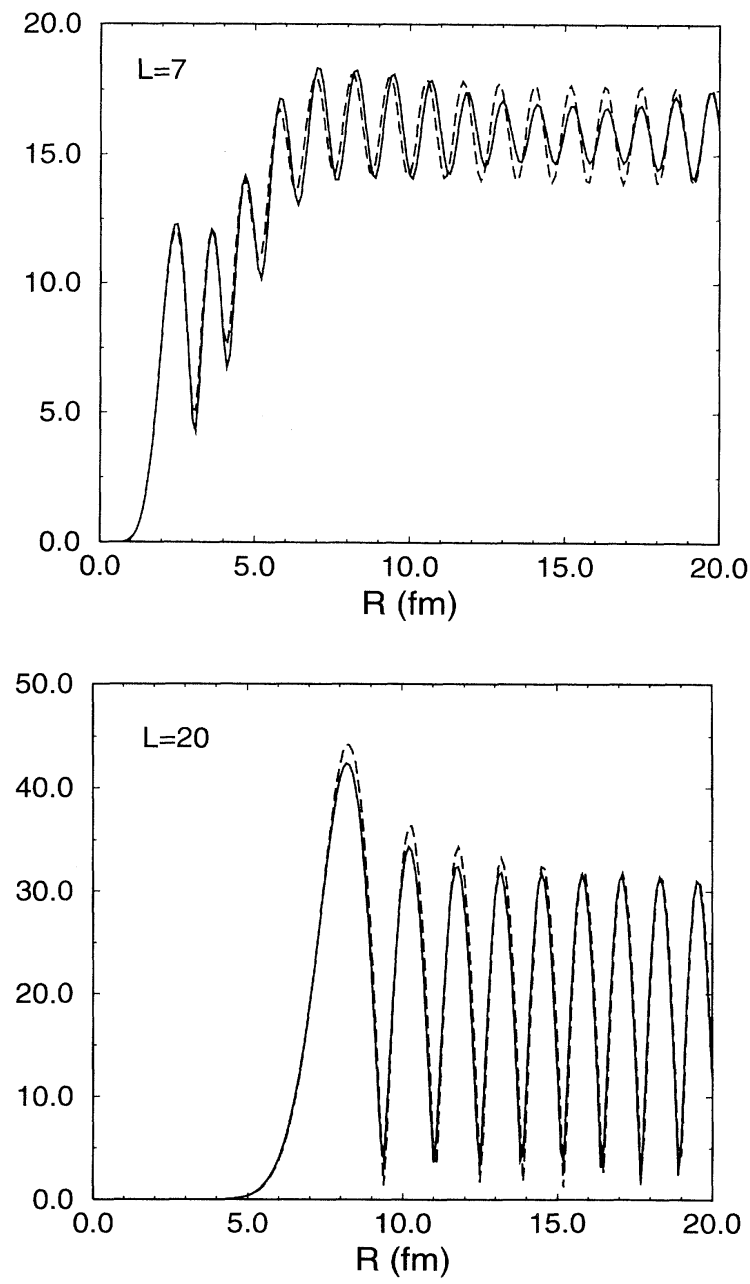

FIG. 6. Moduli of the entrance channel states $\mathcal{X}_{n}^{N}(\mathbf{R})$ in the $L=0,7,13$, and 20 c.m. partial waves, obtained using the WSE model $(N=35, n=3)$ (solid lines), and adiabatic model (dashed lines). 
ing power $i T_{11}$ angular distributions for the selected ${ }^{66} \mathrm{Zn}(d, p){ }^{67} \mathrm{Zn}$ (g.s.; $5 / 2^{-} ; \ell=3$ ) transition at $88.2 \mathrm{MeV}$ incident deuteron energy as a function of the size $N$ of the Weinberg basis. For all $N>15$ there are three open channels; for $N \leq 15$ there are two. As is seen in the figures, for $N>25$ there are only very small changes in the calculated cross section angular distribution. Similarly, for $N \geq 30$, the changes in the calculated $i T_{11}$ angular distribution are small compared with typical error bars on the experimental data at these energies [6]. We note in particular the onset of interference oscillations in the calculated $i T_{11}$ near $\theta \approx 25^{\circ}$ as $N$ is increased.

It is precisely these interference oscillations, the signature of appreciable near-side/far-side interference in the reaction [6], which were not reproduced in adiabatic $(N=1)$ calculations for the transition. In Fig. 8 we demonstrate this point by comparing the $N=35$ WSE calculation above with the predictions of the JohnsonSoper adiabatic result with $N=1$. Also shown are the results of the quasiadiabatic calculation of Ref. [6], but
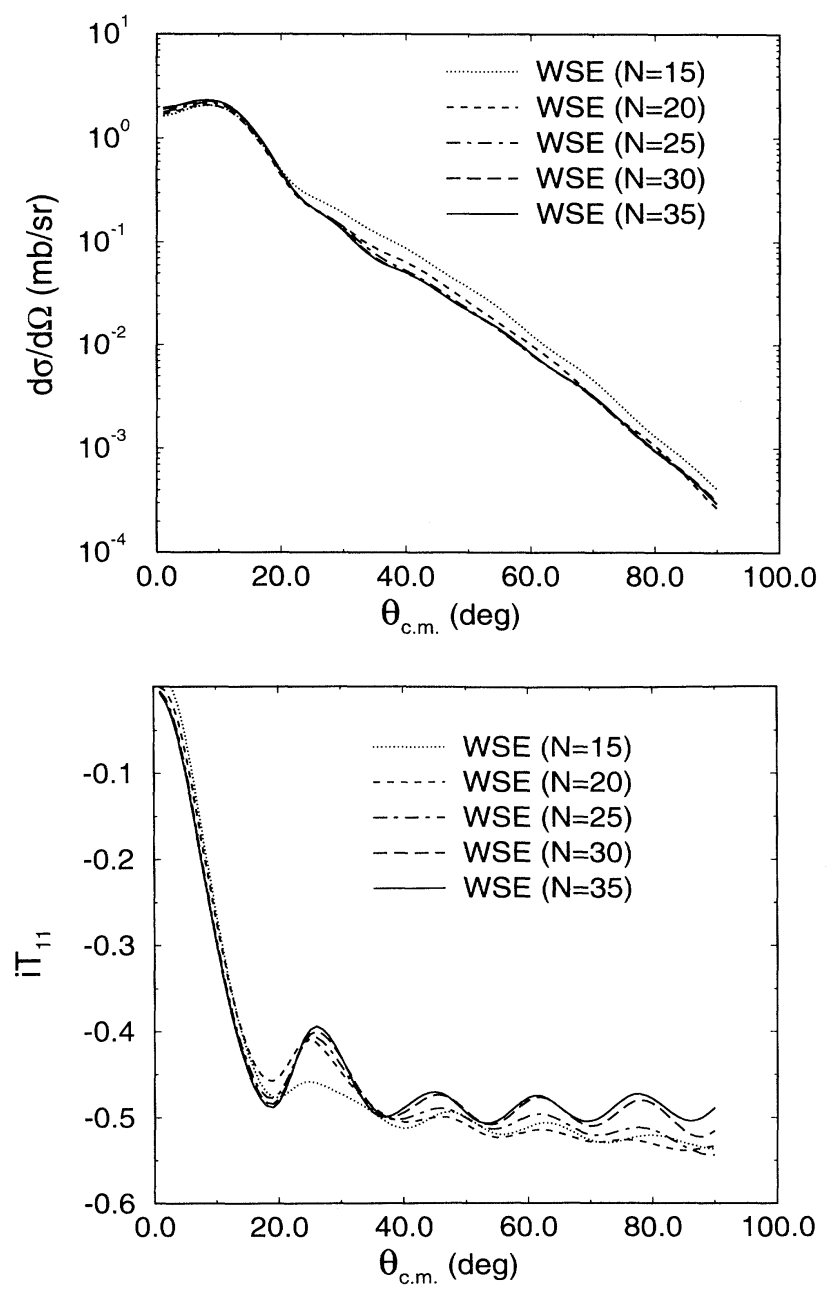

FIG. 7. Calculated cross section and vector analyzing power $i T_{11}$ for the ${ }^{66} \mathrm{Zn}(d, p){ }^{67} \mathrm{Zn}$ (g.s.; $5 / 2^{-} ; \ell=3$ ) transition at $88.2 \mathrm{MeV}$ as a function of the size $N$ of the Weinberg basis used.

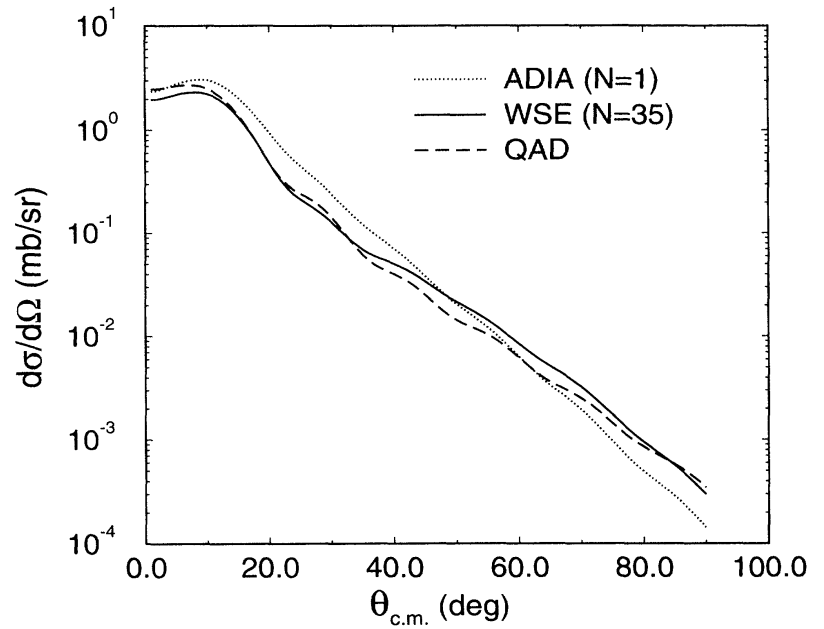

FIG. 8. Comparison of the calculated differential cross section and $i T_{11}$ angular distributions for the ${ }^{66} \mathrm{Zn}(d, p)$ reaction using the $N=35$ WSE model, the adiabatic approximation and the quasiadiabatic model of [6].

in the absence of the nucleon spin-orbit interactions, so as to allow a precise comparison with the WSE calculations. It is clear that the interference pattern in the $i T_{11}$ predicted when using the quasiadiabatic model, so important to reproducing the experimental data in this system, is confirmed by the WSE calculations as arising from the high energy $n-p$ configurations in the entrance channel wave function $(N>1)$ and treated inadequately in the adiabatic approximation.

In the present work we do not include possible contributions from closed $\left|\Delta_{i}^{N}\right\rangle$ channel configurations with $E_{i}=E_{d}-\lambda_{i}<0$. We note from Table I that, for the largest basis calculation presented, $N=35$, the excitation energies of the included states are, in addition to $\lambda_{1}=0.0$, at $\lambda_{2}=15.92 \mathrm{MeV}$, and $\lambda_{3}=56.90 \mathrm{MeV}$. The next lowest configuration has $\lambda_{4}=130.89 \mathrm{MeV}, 74$ $\mathrm{MeV}$ above the last included state. This component is not included in the present calculations. Evidence from the extensive deuteron breakup studies using the CDCC treatment of breakup effects [13] has shown it unnecessary to include, in those calculations, configurations with $n$ - $p$ relative motion wave number $k_{n p}>1.5 \mathrm{fm}^{-1}$, or relative energy of order $90 \mathrm{MeV}$. The contributions from $k_{n p}>1.0 \mathrm{fm}^{-1}$ or relative energy $42 \mathrm{MeV}$ were also found to be very small. While in the present work we use a different $n$ - $p$ continuum basis to that of the CDCC method, the CDCC results, and the calculated contributions from the open channels presented below, suggest that the errors involved in neglecting the closed $\left|\Delta_{i}^{N}\right\rangle$ channels are indeed small.

In Figs. 9 and 10 we investigate the relative importance of the three included open channels on the transfer reaction observables in the calculations with $N=35$. In Fig. 9 we show the calculated differential cross sections obtained when using each component $G_{i}^{N} F_{i}^{(+)}(\mathbf{R})$ of the entrance channel state $\mathcal{X}_{3}^{N}(\mathbf{R})$ individually. While the three contributions interfere when combined to the physical cross section, the figure shows that over most of 


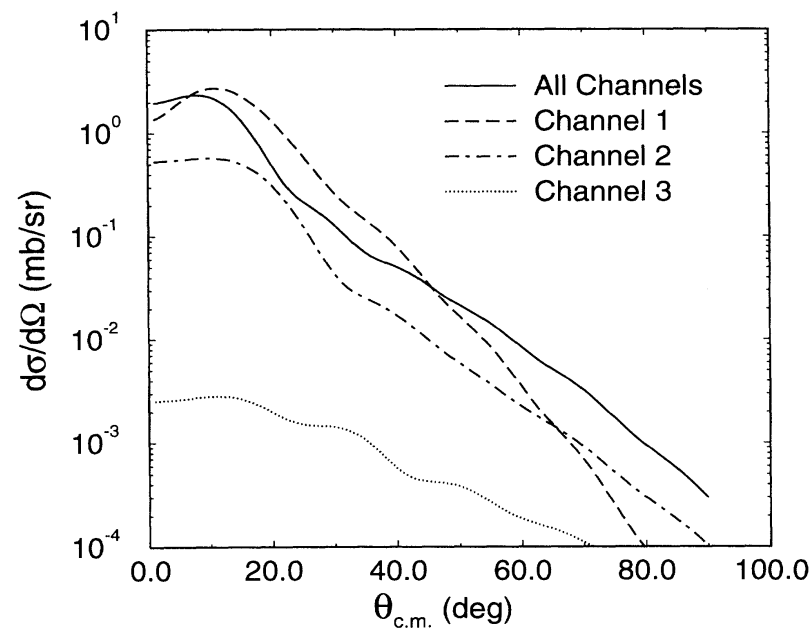

FIG. 9. Comparison of the calculated differential cross section angular distributions due to the $i=1,2$, and 3 open channel contributions to the entrance channel state $\mathcal{X}_{3}^{N}(\mathbf{R})$ of the $N=35$ WSE model.

the angular region, and in particular near $\theta \approx 25^{\circ}$ where the data reveal interference oscillations, the $i=3$ contribution to the transfer amplitude is approximately an order of magnitude smaller than that of the $i=1$ and $i=2$ contributions. In Fig. 10 we show the cumulative effects of including the open channel components of the $N=35, n=3$ coupled-channels calculation of the threebody wave function. The calculated differential cross section and $i T_{11}$ angular distributions are shown when, in the transfer reaction, we include only the $i=1$, the summed $i=1$ and $i=2$, and all three open channel components in the $\mathcal{X}_{n}^{N}(\mathbf{R})$ of Eq. (45). For both observables we note that the contribution from the third open channel, at $56.90 \mathrm{MeV}$ excitation, is relatively small and would indicate that the influence of the next configuration, the closed $\lambda_{4}=130.89 \mathrm{MeV}$ channel, will indeed be negligible for practical purposes.

\section{SUMMARY}

Although formulated some time ago, the Weinberg state technique for the treatment of breakup effects in transfer reactions has not previously been investigated quantitatively. The method, in which the Johnson-Soper adiabatic model appears as the lowest order solution, provides a means to include systematically the leading corrections to that model.

The WSE method is applied to the ${ }^{66} \mathrm{Zn}(d, p){ }^{67} \mathrm{Zn}$ (g.s.) reaction at $88.2 \mathrm{MeV}$. The observables in this reaction have been shown to be particularly sensitive to the treatment of the high energy $n-p$ relative motion components in the $n+p+$ target three-body wave function. We have shown that there are indeed significant corrections to the three-body wave function and to the calculated cross section and vector analyzing power when compared with the Johnson-Soper adiabatic model. The calculations require a Weinberg basis of order 35 states to achieve calculated observables of suitable accuracy for comparison with data in this energy region. Interference oscillations, the signature of appreciable near-side/far-side interference, not reproduced by the adiabatic model, are confirmed by the present calculations as arising from the high energy $n-p$ configurations in the entrance channel wave function, treated inadequately in the adiabatic method.

The aim of the present work was not the reproduction of the available experimental data, but to investigate quantitatively the potential of the WSE method within a realistic model environment. We have shown that, within the WSE formulation, the resulting coupled-channels calculation for the three-body wave function, of relevance to $(d, p)$ reactions near $100 \mathrm{MeV}$, requires only a very small number of physically important open channels to be included. In this work the maximum number of channels used was 3 . Nevertheless, a quite significant number $(N \approx 35)$ of underlying Weinberg states $\left|\phi_{i}\right\rangle$ were required from which to construct these few representative $n-p$ in-
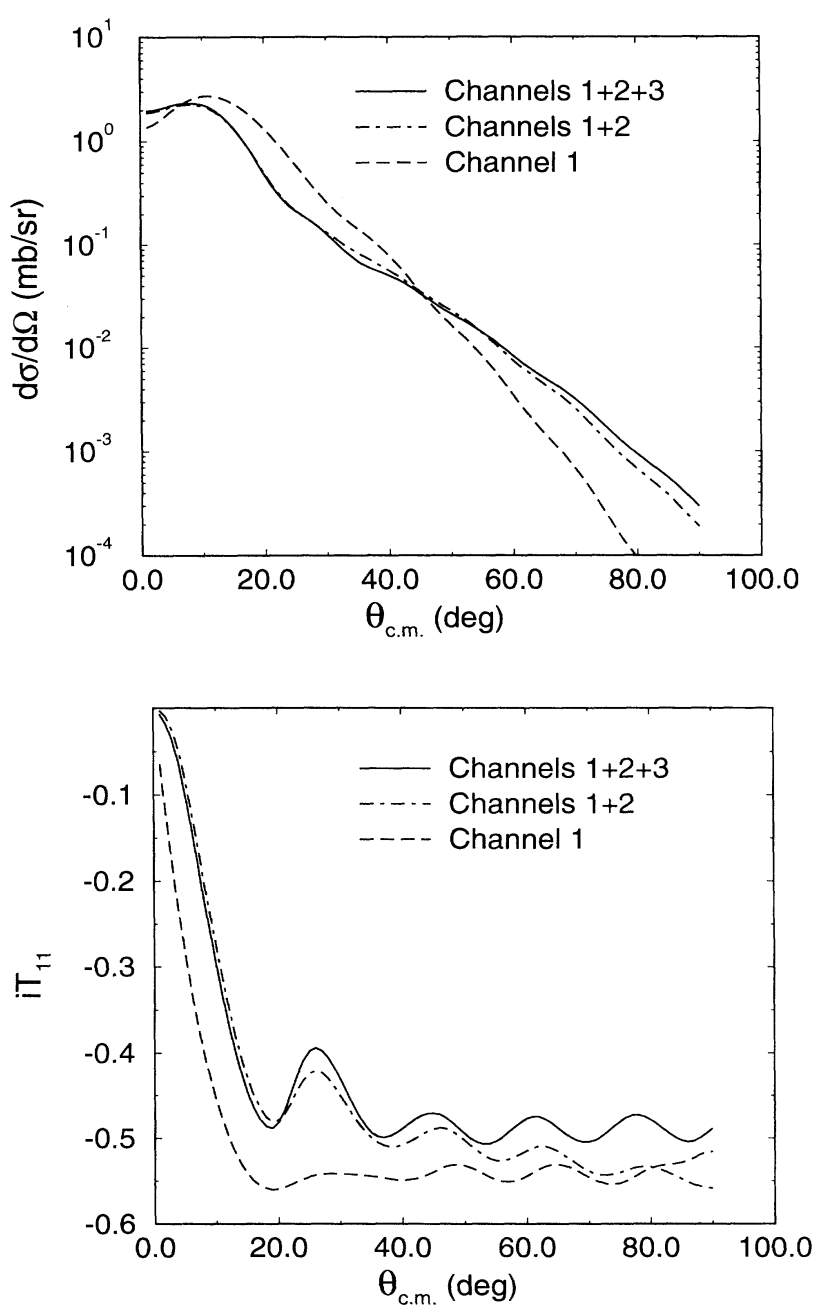

FIG. 10. Comparison of the differential cross section and $i T_{11}$ angular distributions obtained when including the $i=1$ term, the sum of the $(i=1)$ and $(i=2)$ terms, and all terms of the entrance channel state $\mathcal{X}_{3}^{N}(\mathbf{R})$ of the $N=35$ WSE model. 
ternal states $\left|\Delta_{i}^{N}\right\rangle$ and $\left\langle\Omega_{i}^{N}\right|$, required as input to the coupled-channels calculations.

In this sense the Weinberg states might be viewed as an inappropriate expansion basis since, in the final event, the continuum could be represented effectively by a very much smaller function set. However, it is precisely a formal framework to generate this most appropriate set of states, for transfer reactions, which the WSE method addresses. Although a very significant reduction in basis size is achieved, this reduction of the Weinberg basis $\left|\phi_{i}\right\rangle$ to states $\left|\Delta_{i}^{N}\right\rangle$ and $\left\langle\Omega_{i}^{N}\right|$ is independent of any particular reaction transition, target, or projectile energy, depending only on the underlying binding interaction in the composite projectile. As such the numerical reduction need only be carried out once for any particular projectile and transfer interaction, here $V_{n p}$.

It should be noted that the three-body wave function of the WSE method is readily incorporated into finite ranged transfer reaction calculations and the formalism is easily generalized to include noncentral terms in the binding interaction generating the $\left|\phi_{i}\right\rangle$, the $n$ - $p$ tensor interaction in the case of the deuteron. Zero-range calculations were used here only to allow comparison with the earlier quasiadiabatic results, where the calculated three-body wave function is not expressed as a product of functions of c.m. and internal projectile coordinates, and does not lend itself so readily to such a finite range generalization.

It would be interesting and straightforward to extend the analysis to other systems. The $\left(d,{ }^{6} \mathrm{Li}\right)$ reaction has been used extensively for alpha particle spectroscopy studies, with $E_{d} \approx 100 \mathrm{MeV}$, using the DWBA. The WSE method would provide a natural framework within which to investigate the magnitude of breakup corrections in this system. In particular, the technique should also find application when the rapidly increasing database on reactions induced by light neutron-rich radioactive nuclei, e.g., ${ }^{11} \mathrm{Be}$, with large breakup channel effects, is extended to include single-nucleon or cluster transfer reaction data.

\section{ACKNOWLEDGMENTS}

The support of the Science and Engineering Research council (U.K.) through Grants No. GR/G 0109.6 and No. GR/H 2402.0 (for R.C.J. and J.A.T.) and of financial support from the Algerian Government (for A.L.) are gratefully acknowledged.
[1] N. Austern, Y. Iseri, M. Kamimura, M. Kawai, G. Rawitscher, and M. Yahiro, Phys. Rep. 154, 125 (1987).

[2] R. C. Johnson and P. J. R. Soper, Phys. Rev. C 1, 976 (1970).

[3] J. D. Harvey and R. C. Johnson, Phys. Rev. C 3, 636 (1971); H. Ohnuma, T. Suehiro, M. Sekiguchi, and S. Yamada, J. Phys. Soc. Jpn. 36, 1245 (1974).

[4] G. L. Wales and R. C. Johnson, Nucl. Phys. A274, 168 (1976).

[5] E. J. Stephenson, R. C. Johnson, J. A. Tostevin, V. R. Cupps, J. D. Brown, C. C. Foster, J. A. Gering, W. P. Jones, D. A. Low, D. W. Miller, H. Nann, C. Olmer, P. Schwandt, J. W. Seubert, and S. W. Wissink, Phys. Lett. B 171,358 (1986); R. C. Johnson, E. J. Stephenson, and J. A. Tostevin, Nucl. Phys. A505, 26 (1989).

[6] E. J. Stephenson, A. D. Bacher, G. P. A. Berg, V. R. Cupps, C. C. Foster, N. Hodiwalla, P. C. Li, J. Lisantti, D. A. Low, D. W. Miller, C. Olmer, A. K. Opper, B. K. Park, R. Sawafta, S. W. Wissink, J. A. Tostevin, D. A. Coley, and R. C. Johnson, Phys. Rev. C 42, 2562 (1990).
[7] H. Amakawa, N. Austern, and C. M. Vincent, Phys. Rev. C 29, 699 (1984).

[8] R. C. Johnson and P. C. Tandy, Nucl. Phys. A235, 56 (1974).

[9] W. F. Junkin and F. Villars, Ann. Phys. (N.Y.) 45, 93 (1967); 51, 68 (1969); N. Austern and K. C. Richards, ibid. 49, 309 (1968); S. Mukherjee, Nucl. Phys. A118, 423 (1968).

[10] S. Weinberg, Phys. Rev. 131, 440 (1963).

[11] R. G. Newton, Scattering Theory of Waves and Particles (McGraw-Hill, New York, 1966), p. 282.

[12] F. D. Becchetti, Jr. and G. W. Greenlees, Phys. Rev. 182, 1190 (1969).

[13] M. Kamimura, M. Yahiro, Y. Iseri, H. Kameyama, Y. Sakuragi, and M. Kawai, Prog. Theor. Phys. Suppl. 89, 1 (1986).

[14] I. J. Thompson, computer program FRESCO, University of Surrey (unpublished); Comput. Phys. Rep. 7, 167 (1988).

[15] M. Igarashi, M. Toyama, and N. Kishida, Computer Program TWOFNR (private communication). 